6-8-2021

\title{
Two Different Classes of Shrinkage Estimators for the Scale Parameter of the Rayleigh Distribution
}

\author{
Talha Omer \\ University of Veterinary and Animal Sciences, Lahore, talha.omer@uvas.edu.pk \\ Zawar Hussain \\ Cholistan University of Veterinary \& Animal Sciences \\ Muhammad Qasim \\ University of Veterinary and Animal Sciences, Lahore, muhammad.qasim@ju.se \\ Said Farooq Shah \\ University of Peshawar \\ Akbar Ali Khan \\ Directorate of Higher Education KP, Peshawar, Pakistan
}

Follow this and additional works at: https://digitalcommons.wayne.edu/jmasm

Part of the Applied Statistics Commons, Social and Behavioral Sciences Commons, and the Statistical Theory Commons

\section{Recommended Citation}

Omer, Talha; Hussain, Zawar; Qasim, Muhammad; Shah, Said Farooq; and Khan, Akbar Ali (2021) "Two Different Classes of Shrinkage Estimators for the Scale Parameter of the Rayleigh Distribution," Journal of Modern Applied Statistical Methods: Vol. 19 : Iss. 1 , Article 8.

DOI: $10.22237 / \mathrm{jmasm} / 1608553440$

Available at: https://digitalcommons.wayne.edu/jmasm/vol19/iss1/8

This Emerging Scholar is brought to you for free and open access by the Open Access Journals at DigitalCommons@WayneState. It has been accepted for inclusion in Journal of Modern Applied Statistical Methods by an authorized editor of DigitalCommons@WayneState. 


\section{Two Different Classes of Shrinkage Estimators for the Scale Parameter of the Rayleigh Distribution}

\section{Cover Page Footnote}

The authors would like to thank the Editor and the anonymous referee for their valuable and constructive suggestions that definitely improved the presentation and quality of this article. 


\section{EMERGING SCHOLAR}

\section{Two Different Classes of Shrinkage Estimators for the Scale Parameter of the Rayleigh Distribution}

Talha Omer

University of Veterinary and

Animal Science

Lahore, Pakistan

\section{Zawar Hussain}

Cholistan University of

Veterinary \& Animal Sciences

Bahawalpur, Pakistan
Muhammad Qasim

University of Veterinary and

Animal Science

Lahore, Pakistan
Said Farooq Shah

University of Peshawar

Peshawar, Pakistan

\author{
Akbar Ali Khan \\ Directorate of Higher Education KP \\ Peshawar, Pakistan
}

Shrinkage estimators are introduced for the scale parameter of the Rayleigh distribution by using two different shrinkage techniques. The mean squared error properties of the proposed estimator have been derived. The comparison of proposed classes of the estimators is made with the respective conventional unbiased estimators by means of mean squared error in the simulation study. Simulation results show that the proposed shrinkage estimators yield smaller mean squared error than the existence of unbiased estimators.

Keywords: $\quad$ Rayleigh distribution, scale parameter, shrinkage estimator, mean squared error

\section{Introduction}

Unbiased estimators are commonly used for making inferences about an unknown descriptive parameter(s) of a population. In cases, when unbiased estimators possess larger variances, biased estimators with smaller mean square error (MSE) are preferred. Searls (1964), Thompson (1968), Mehta and Srinivasan (1971), Das (1975), Srivastava et al. (1980), Rao and Singh (1982), Bhatnagar (1986), Singh and Katyar (1988), Rytgaard (1990), Jani (1991), Kourouklis (1994), Singh and Singh (1997), Singh and Shukla (2003), Singh and Saxena (2003), Prakash et al.

doi: 10.22237/jmasm/1608553440 | Accepted: October 4, 2018; Published: June 8, 2021.

Correspondence: Muhammad Qasim, qasim.stat@gmail.com 


\section{OMER ET AL}

(2006), Prakash (2009), Ebegil and Ozdemir (2016) and many others proposed numerous biased estimators as an alternative to the unbiased estimator.

Rayleigh distribution was introduced by Rayleigh (1880) after facing a problem in acoustics. It has a wide range of applications in almost every field of science where the phenomena of interest assume positive value. In this study, we deal with two different classes of shrinkage-type estimators for the scale parameter of the Rayleigh distribution. The proposed classes of the estimators will be compared with the usual unbiased estimators in terms of MSE by deriving optimal conditions. For this purpose, a simulation study will be conducted for numerical comparisons.

\section{The Class of Shrinkage Estimators}

Jani (1991) and Singh and Singh (1997) proposed two different shrinkage estimator classes for the scale parameter of the exponential and normal distributions. Shrinkage estimator class of Jani for the scale parameter of the exponential distribution is defined in Equation (1):

$$
T_{p}=\theta_{0}\left[1+k\left(\frac{\theta_{0}}{\hat{\theta}}\right)^{p}\right]
$$

where $\theta_{0}$ is an a priori value of the parameter $\theta, k$ is a shrinkage factor that minimizes the MSE value, $p$ is a nonzero real number, and $\hat{\theta}_{0}$ is the unbiased estimator of the parameter $\theta$. Singh and Singh (1997) considered the estimation problem of population variance by adapting the same class defined in Equation (1) for a normal population. The class of estimators by Singh and Singh is given in Equation (2):

$$
\hat{\sigma}_{(p)}^{2}=\sigma_{0}^{2}\left[1+w\left(\frac{s^{2}}{\sigma_{0}^{2}}\right)^{p}\right]
$$

where $\sigma_{0}^{2}$ is an a priori value of the parameter $\sigma^{2}, w$ is a shrinkage factor that minimizes the MSE value, $p$ is a nonzero real number, and $s^{2}$ is an unbiased estimator of the parameter $\sigma^{2}$.

Similarly, Ebegil and Ozdemir (2016) proposed biased estimators for the shape parameter of the classical Pareto distribution using two different shrinkage 


\section{SHRINKAGE ESTIMATORS FOR RAYLEIGH DISTRIBUTION}

techniques. One of the two classes proposed by Singh and Singh (1997) is given below:

$$
\beta_{(p)}^{*}=\beta_{0}+\left(\hat{\beta}-\beta_{0}\right) k_{(p)}
$$

where

$$
k_{(p)}=(n-1)^{p} \frac{(n+p-1) !}{(n+2 p-1) !}
$$

and $p$ is a nonzero real number.

Next, a shrinkage type of estimators will be developed having smaller MSEs as compared to the unbiased estimator for the scale parameter of Rayleigh distribution. Specifically, we develop the estimators in line to Equations (1)-(3).

Let $X$ be a random variable following the Rayleigh distribution. The probability density function (pdf) of $X$ is given by

$$
\mathrm{f}(x)=\frac{x}{\theta} e^{\frac{-x^{2}}{2 \theta}} ; x \geq 0, \theta>0,
$$

where, $\theta$ is the scale parameter of the Rayleigh distribution. The maximum likelihood estimator (MLE) for the scale parameter $\theta$ of the Rayleigh distribution is

$$
\hat{\theta}_{\mathrm{MLE}}=\frac{\sum_{i=1}^{n} X_{i}^{2}}{2 n}=\frac{Y}{2 n} .
$$

It is evident that $Y$ has $\mathrm{G}(n, 2 \theta)$ and the pdf of $Y$ is given by

$$
\mathrm{f}(y)=\frac{1}{\Gamma(n)(2 \theta)^{n}} y^{n-1} e^{\frac{-y}{2 \theta}}
$$

The estimator $\hat{\theta}_{\mathrm{MLE}}$ is unbiased having variance

$$
\operatorname{Var}\left(\hat{\theta}_{\mathrm{MLE}}\right)=\frac{\theta^{2}}{n}
$$




\section{OMER ET AL}

\section{The Proposed Shrinkage-Type Estimators}

Two different classes of shrinkage-type estimators for the scale parameter of the Rayleigh distribution are derived and discussed below.

Theorem 1. Following Singh and Singh (1997), the class of shrinkage estimators for the scale parameter of Rayleigh distribution is given by

$$
\theta_{p}^{*}=\theta_{0}+k_{(p)}\left(\hat{\theta}_{\mathrm{MLE}}-\theta_{0}\right)
$$

The MSE and bias of $\theta_{p}^{*}$ are defined in Equation (7) and Equation (8), respectively:

$$
\begin{gathered}
\operatorname{MSE}\left(\theta_{p}^{*}\right)=\theta^{2}\left[\frac{k_{(p)}^{2}}{n}+\left(k_{(p)}-1\right)^{2}\left(1-\lambda^{-1}\right)^{2}\right], \\
\operatorname{Bias}\left(\theta_{p}^{*}\right)=\left(1-k_{(p)}\right)\left(\theta_{0}-\theta\right),
\end{gathered}
$$

where

$$
k_{(p)}=\frac{(n+p-1) ! n^{p}}{(n+2 p-1) !} \quad \text { and } \quad \lambda=\frac{\theta}{\theta_{0}} .
$$

Proof. $\quad$ From Equation (2), we have

$$
\theta_{p}^{*}=\theta_{0}\left[1+k\left(\frac{\left(\hat{\theta}_{\mathrm{MLE}}\right)^{p}}{\theta_{0}^{p}}\right)\right]
$$

Following equations will be used to derive the MSE of the class of estimator in Equation (9).

$$
\mathrm{E}\left[\left(\hat{\theta}_{\mathrm{MLE}}\right)^{j p}\right]=K_{1(j p)} \theta^{j p},(j=1,2)
$$




\section{SHRINKAGE ESTIMATORS FOR RAYLEIGH DISTRIBUTION}

and

$$
K_{1(j p)}=\frac{(n+j p-1) !}{n^{j p}(n-1) !} .
$$

The MSE of the $\theta_{p}^{*}$ estimator is defined as

$$
\operatorname{MSE}\left(\theta_{p}^{*}\right)=\mathrm{E}\left[\theta_{p}^{*}-\theta\right]^{2}
$$

Using Equations (9)-(11) in Equation (12), after necessary calculations, we get

$$
\operatorname{MSE}\left(\theta_{p}^{*}\right)=\theta^{2}\left[\left(1-\lambda^{-1}\right)^{2}+k^{2} \lambda^{2 p-2} K_{1(2 p)}+2 k K_{1(p)} \lambda^{p-2}(1-\lambda)\right] .
$$

To obtain optimum value of $k$, Equation (13) is differentiated with respect to $k$ and set equal to zero. After simplification, we get

$$
k=\frac{K_{(1 p)}}{K_{(2 p)}} \lambda^{-p}(\lambda-1)
$$

which minimizes the MSE of Equation (13). After substituting the required values in Equation (14), $k$ is defined as

$$
k=k(p)\left(\frac{\theta}{\theta_{0}}\right)^{p}\left(\frac{\theta}{\theta_{0}}-1\right)
$$

The shrinking parameter $k$ is obtained as a function of the parameter $\theta$. In practice, it is impossible to obtain a true value of the parameter $\theta$. Therefore, the unknown parameter of Equation (15) is replaced by its unbiased estimator. So, the estimator for $k$ is obtained as

$$
\hat{k}=k(p)\left(\frac{\hat{\theta}_{\mathrm{MLE}}}{\theta_{0}}\right)^{p}\left(\frac{\hat{\theta}_{\mathrm{MLE}}}{\theta_{0}}-1\right)=k(p)\left(\frac{\hat{\theta}_{\mathrm{MLE}}}{\theta_{0}}\right)^{p}\left(\frac{\hat{\theta}_{\mathrm{MLE}}-\theta_{0}}{\theta_{0}}\right) .
$$




\section{OMER ET AL}

After necessary adjustment, the class of estimators for the scale parameter of the Rayleigh distribution is derived as given in Equation (6).

The MSE of the $\theta^{*}$ estimator is derived by using Equation (16) from Equation (13) as follows:

$$
\operatorname{MSE}\left(\theta_{p}^{*}\right)=k_{(p)}^{2} \frac{\theta^{2}}{n}+\left(k_{(p)}-1\right)^{2}\left(\theta-\theta_{0}\right)^{2}
$$

Simplification of Equation (17) can be reduced to Equation (7).

The bias of $\theta_{p}^{*}$ is computed as

$$
\operatorname{Bias}\left(\theta_{p}^{*}\right)=\mathrm{E}\left(\theta_{p}^{*}\right)-\theta
$$

Taking the expectation of Equation (12) and using Equations (10) and (11) in Equation (18), we get Equation (8), which completes the proof.

\section{The Relative Efficiency of the First Estimator}

The relative efficiency of the class of estimators $\theta_{p}^{*}$ with respect to $\hat{\theta}_{\mathrm{MLE}}$ is obtained as

$$
\text { Relative Efficiency }=\frac{\operatorname{MSE}\left(\theta_{p}^{*}\right)}{\operatorname{Var}\left(\hat{\theta}_{\mathrm{MLE}}\right)}=k_{(p)}^{2}+(n-2)+n\left(k_{(p)-1}\right)^{2}\left(1-\lambda^{-1}\right)^{2}
$$

Hence, it is clear that $\operatorname{MSE}\left(\theta_{p}^{*}\right) \leq \operatorname{Var}\left(\hat{\theta}_{\mathrm{MLE}}\right)<1$.

\section{Case 1}

Consider $p=1$ in Equation (6). Then an estimator is obtained as

$$
\theta_{1}^{*}=\theta_{0}+\frac{n}{n+1}\left(\hat{\theta}_{\mathrm{MLE}}-\theta_{0}\right)
$$

The MSE of this estimator is calculated as 


$$
\operatorname{MSE}\left(\theta_{1}^{*}\right)=\theta^{2}\left[\frac{n^{2}}{(n+1)}+\frac{n}{(n+1)}\left(1-\lambda^{-1}\right)^{2}\right]
$$

The relative efficiency of the estimator $\theta_{1}^{*}$ with respect to $\hat{\theta}_{\mathrm{MLE}}$ is

$$
\frac{\operatorname{MSE}\left(\theta_{1}^{*}\right)}{\operatorname{Var}\left(\hat{\theta}_{\mathrm{MLE}}\right)}=n^{2}\left[\frac{n}{(n+1)}+\frac{1}{(n+1)}\left(1-\lambda^{-1}\right)^{2}\right]
$$

It is clear that $\theta_{1}^{*}$ is better than $\hat{\theta}_{\mathrm{MLE}}$ if

$$
\frac{\operatorname{MSE}\left(\theta_{1}^{*}\right)}{\operatorname{Var}\left(\hat{\theta}_{\mathrm{MLE}}\right)} \leq 1 \text { or }\left(1-\lambda^{-1}\right)^{2} \leq \frac{1-n^{2}}{n^{2}}
$$

when $n$ tends to $\infty$, the above inequality reduces to $0 \leq \lambda \leq 1$.

\section{Case 2}

Suppose $p=2$ in Equation (6), then the following estimator is obtained:

$$
\theta_{2}^{*}=\theta_{0}+\frac{n^{2}}{(n+3)}\left(\hat{\theta}_{\mathrm{MLE}}-\theta_{0}\right)
$$

The MSE of the estimator $\theta_{2}^{*}$ is defined as

$$
\operatorname{MSE}\left(\theta_{2}^{*}\right)=\theta^{2}\left[n\left(\frac{n^{4}}{(n+3)^{2}(n+2)^{2}}\right)+\frac{n^{2}-(n+3)(n+2)}{(n+3)(n+2)}\left(1-\lambda^{-1}\right)^{2}\right]
$$

The relative efficiency of the estimator $\theta_{2}^{*}$ with respect to $\hat{\theta}_{\mathrm{MLE}}$ is

$$
\frac{\operatorname{MSE}\left(\theta_{2}^{*}\right)}{\operatorname{Var}\left(\hat{\theta}_{\mathrm{MLE}}\right)}=\left[n\left(\frac{n^{2}}{(n+3)(n+2)}\right)^{2}+\left(\frac{n^{2}}{(n+3)(n+2)}-1\right)^{2}\left(1-\lambda^{-1}\right)^{2}\right] .
$$




\section{OMER ET AL}

The estimator $\theta_{2}^{*}$ is more efficient than $\hat{\theta}_{\mathrm{MLE}}$ if and only if

$$
\begin{gathered}
\frac{\operatorname{MSE}\left(\theta_{2}^{*}\right)}{\operatorname{Var}\left(\hat{\theta}_{\mathrm{MLE}}\right)}<1 \\
\frac{1}{1+\sqrt{\frac{2 n^{2}+5 n+6}{n(5 n+6)}}} \leq \lambda \leq \frac{1}{1-\sqrt{\frac{2 n^{2}+5 n+6}{n(5 n+6)}}}
\end{gathered}
$$

Furthermore, when $n$ is very large, i.e., $n \rightarrow \infty$, then

$$
\left(1-\lambda^{-1}\right)^{2} \leq \frac{\left(2+\frac{5}{n}+\frac{6}{n^{2}}\right)}{\left(5+\frac{6}{n}\right)}
$$

reduces to $0.61 \leq \lambda \leq 1.63$.

Theorem 2. Following Jani (1991), the class of shrinkage estimators for the scale parameter of the Rayleigh distribution is defined as

$$
\theta_{p}^{* *}=\theta_{0}+w_{(p)}\left(\hat{\theta}_{\mathrm{MLE}}-\theta_{0}\right)
$$

where

$$
w_{(p)}=n^{-p} \frac{(n-p-1) !}{(n-2 p-1) !} .
$$

The bias of $\theta_{p}^{* *}$ is given as

$$
\operatorname{Bias}\left(\theta_{p}^{* *}\right)=\mathrm{E}\left(\theta_{p}^{* *}\right)-\theta=\left(1-w_{(p)}\right)\left(\theta-\theta_{0}\right)
$$

The MSE of $\theta_{p}^{* *}$ is given below: 


\section{SHRINKAGE ESTIMATORS FOR RAYLEIGH DISTRIBUTION}

$$
\operatorname{MSE}\left(\theta_{p}^{* *}\right)=\theta^{2}\left[\frac{w_{(p)}^{2}}{n}+\left(w_{(p)}-1\right)^{2}\left(1-\lambda^{-1}\right)^{2}\right]
$$

Proof. $\quad$ From Equation (1), the class of shrinkage-type estimators for the scale parameter of the Rayleigh distribution is defined as

$$
\theta_{p}^{* *}=\theta_{0}\left[1+w\left(\frac{\theta_{0}}{\hat{\theta}_{\mathrm{MLE}}}\right)^{p}\right]
$$

Here,

$$
\mathrm{E}\left(\hat{\theta}_{\mathrm{MLE}}\right)^{-j p}=W_{(j p)} \theta^{-j p}
$$

and

$$
W_{(j p)}=\frac{(n-j p-1) ! n^{j p}}{(n-1) !} .
$$

The expression in Equations (24) and (25) are used to calculate the MSE of $\theta_{p}^{* *}$. The MSE of $\theta_{p}^{* *}$ is derived as

$$
\operatorname{MSE}\left(\theta_{p}^{* *}\right)=\mathrm{E}\left(\theta_{p}^{* *}-\theta\right)^{2}
$$

Using Equations (23)-(25) in Equation (26), the MSE of $\theta_{p}^{* *}$ is obtained as

$$
\operatorname{MSE}\left(\theta_{p}^{* *}\right)=\theta^{2}\left[\left(1+\lambda^{-1}\right)^{2}+w^{2} W_{(2 p)} \lambda^{-2(p+1)}+2 w W_{(1 p)} \lambda^{(-p-1)}\left(\lambda^{-1}-1\right)\right] .
$$

Differentiating Equation (27) with respect to $w$ and equating to zero, we find an optimum value of $w$ as given below: 


\section{OMER ET AL}

$$
w=w_{(p)}\left(\frac{\theta}{\theta_{0}}\right)^{p}\left(\frac{\theta}{\theta_{0}}-1\right)
$$

where

$$
w_{(p)}=\frac{W_{(1 p)}}{W_{(2 p)}} .
$$

On substituting $W_{(1 p)}$ and $W_{(2 p)}$ in Equation (29), we get

$$
w_{(p)}=\frac{(n-p-1) !}{(n-2 p-1) ! n^{p}} .
$$

Now, using Equation (30) in Equation (28), we get

$$
w=\left(\frac{(n-p-1) !}{(n-2 p-1) !\left(n^{p}\right)}\right) \frac{\theta^{p}}{\theta_{0}^{p}}\left(\frac{\theta}{\theta_{0}}-1\right) .
$$

The shrinkage parameter $w$ is obtained as a function of the unknown parameter $\theta$. So, the unknown parameter in Equation (31) is replaced by its unbiased estimator. So, the $\hat{w}$ estimator for $w$ is computed as

$$
\hat{w}=\left(\frac{(n-p-1) !}{(n-2 p-1) !\left(n^{p}\right)}\right) \frac{\hat{\theta}_{\mathrm{MLE}}^{p}}{\theta_{0}^{p}}\left(\frac{\hat{\theta}_{\mathrm{MLE}}^{p}}{\theta_{0}}-1\right) .
$$

Finally, substituting Equation (32) into Equation (23), the class of estimators for the scale parameter of the Rayleigh distribution is obtained as

$$
\theta_{p}^{* *}=\theta_{0}+w_{(p)}\left(\hat{\theta}_{\mathrm{MLE}}-\theta_{0}\right)
$$

Similarly, from Equations (25), (27), and (30), the MSE of $\theta_{p}^{* *}$ is obtained as 


$$
\operatorname{MSE}\left(\theta_{p}^{*}\right)=w_{(p)}^{2} \frac{\theta^{2}}{n}+\left(w_{(p)}-1\right)^{2}\left(\theta-\theta_{0}\right)^{2}
$$

After simplification, Equation (33) can be reduced to Equation (22).

The bias of $\theta_{p}^{* *}$ is obtained as

$$
\operatorname{Bias}\left(\theta_{p}^{* *}\right)=\mathrm{E}\left(\theta_{p}^{* *}\right)-\theta
$$

By taking the expectation of Equation (23) and substituting Equations (23) and (24) in Equation (33), Equation (33) reduced to Equation (21).

\section{The Relative Efficiency of the Second Estimator}

The relative efficiency of $\theta_{p}^{* *}$ with respect to $\hat{\theta}_{\mathrm{MLE}}$ is derived as

$$
\text { Relative Efficiency }=\frac{\operatorname{MSE}\left(\theta_{p}^{* *}\right)}{\operatorname{Var}\left(\hat{\theta}_{\mathrm{MLE}}\right)}=\left[w_{(p)}^{2}+n\left(w_{(p)}-1\right)^{2}\left(1-\lambda^{-1}\right)^{2}\right]
$$

\section{Case 1}

Suppose $p=1$ in Equation (20); the MSE of the estimator for the scale parameter can be obtained as

$$
\theta_{1}^{* *}=\theta_{0}\left[1+\frac{n-2}{n}(\hat{\theta}-\theta)\right]
$$

The MSE of the estimator in Equation (34) is defined as

$$
\operatorname{MSE}\left(\theta_{1}^{* *}\right)=\theta^{2}\left[\frac{(n-2)^{2}}{n^{3}}+\frac{4}{n^{2}}\left(1-\lambda^{-1}\right)^{2}\right]
$$

The relative efficiency of $\theta_{1}^{* *}$ with respect to $\hat{\theta}_{\mathrm{MLE}}$ is given by the following expression: 


\section{OMER ET AL}

$$
\frac{\operatorname{MSE}\left(\theta_{1}^{* *}\right)}{\operatorname{Var}\left(\hat{\theta}_{\mathrm{MLE}}\right)}=\frac{(n-2)^{2}}{n^{2}}+\frac{4}{n}\left(1-\lambda^{-1}\right)^{2}
$$

The $\theta_{1}^{* *}$ estimator will be more efficient than $\hat{\theta}_{\mathrm{MLE}}$ if

$$
\frac{\operatorname{MSE}\left(\theta_{1}^{* *}\right)}{\operatorname{Var}\left(\hat{\theta}_{\mathrm{MLE}}\right)}<1
$$

which gives

$$
\frac{(n-2)^{2}}{n^{2}}+\frac{4}{n}\left(1-\lambda^{-1}\right)^{2} \leq 1
$$

After simplification, the above inequality becomes

$$
\left(1-\lambda^{-1}\right)^{2} \leq \frac{2 n-3}{4}
$$

For $n \rightarrow \infty$, this inequality reduces to $0<\lambda<1$.

\section{Case 2}

By considering $p=2$ in Equation (20), an estimator for the scale parameter is obtained as

$$
\theta_{2}^{* *}=\theta_{0}+\frac{(n-3)(n-2)}{n^{2}}\left(\hat{\theta}_{\mathrm{MLE}}-\theta_{0}\right)
$$

The MSE of the $\theta_{2}^{* *}$ estimator is given by

$$
\operatorname{MSE}\left(\theta_{2}^{* *}\right)=\theta^{2}\left[n^{-1}((n-3)(n-4))^{2}+\left(\frac{(n-3)(n-4)}{n^{2}}-1\right)^{2}\left(1-\lambda^{-1}\right)^{2}\right]
$$

The relative efficiency of $\theta_{2}^{* *}$ with respect to $\hat{\theta}_{\mathrm{MLE}}$ is obtained as 


\section{SHRINKAGE ESTIMATORS FOR RAYLEIGH DISTRIBUTION}

$$
\frac{\operatorname{MSE}\left(\theta_{2}^{* *}\right)}{\operatorname{Var}\left(\hat{\theta}_{\mathrm{MLE}}\right)}=\left[n^{-1}((n-3)(n-4))^{2}+\left(\frac{(n-3)(n-4)}{n^{2}}-1\right)^{2}\left(1-\lambda^{-1}\right)^{2}\right]
$$

It is clear that $\theta_{2}^{* *}$ is better than $\hat{\theta}_{\mathrm{MLE}}$ if

$$
\frac{\operatorname{MSE}\left(\theta_{2}^{* *}\right)}{\operatorname{Var}\left(\hat{\theta}_{\mathrm{MLE}}\right)} \leq 1
$$

This gives

$$
n\left(1-\frac{(n-3)(n-4)}{n^{2}}\left(1-\lambda^{-1}\right)^{2}\right) \leq\left(1+\frac{(n-3)(n-4)}{n^{2}}\right)
$$

After simplification, we get

$$
\frac{1}{1+\sqrt{\frac{2 n^{2}-7 n+12}{n(7 n-12)}}} \leq \lambda \leq \frac{1}{1-\sqrt{\frac{2 n^{2}-7 n+12}{n(7 n-12)}}}
$$

When $n$ is very large $(n \rightarrow \infty)$, the inequality reduces to $0.65 \leq \lambda \leq 2.14$.

Note. It can be identified that the class of estimators proposed by Jani (1991) is directly related to the Singh and Singh (1997) estimator for the scale parameter of the Rayleigh distribution. This relationship is expressed as $k_{(-p)}=w_{(p)}$.

\section{Numerical Study}

The percent relative bias and MSE are considered as performance criteria to judge the performance of the proposed estimation. A relative bias can be calculated by dividing Equation (11) into Equation (21). The relative bias is given in Equation (35):

$$
\frac{\operatorname{Bias}\left(\theta_{p}^{*}\right)}{\operatorname{Bias}\left(\theta_{p}^{* *}\right)}=\frac{1-k_{(p)}}{1-w_{(p)}}
$$




\section{OMER ET AL}

The values of relative bias are obtained by means of Equation (35) for $n=10,15$, 20, 25, and 50 and for different values of $p$. These values are shown in Table 1. It is observed that the biases of $\theta_{p}^{* *}$ are smaller (larger) than those of $\theta_{p}^{*}$ when $p$ is negative (positive). Moreover, it is seen that the relative bias decreases (increases) when the sample size increases and $p$ is negative (positive). However, it can be noted that the $\theta_{p}^{* *}$ have a smaller bias when the value of $p$ is negative, while they have a larger bias when the value of $p$ is positive.

Table 1. The relative bias of $\theta_{p}^{*}$ relative to $\theta_{p}^{* *}$ for different $n$ and $p$ values

\begin{tabular}{rrrrrr} 
& \multicolumn{5}{c}{ Sample size } \\
\cline { 2 - 6 } $\boldsymbol{p}$ & $\mathbf{1 0}$ & $\mathbf{1 5}$ & $\mathbf{2 0}$ & $\mathbf{2 5}$ & $\mathbf{5 0}$ \\
\hline-1.0 & 2.2000 & 2.1333 & 2.1000 & 2.0800 & 2.0400 \\
-0.5 & 5.1860 & 5.1214 & 5.0901 & 5.0717 & 5.0354 \\
0.5 & 0.1928 & 0.1952 & 0.1964 & 0.1971 & 0.1985 \\
1.0 & 0.4545 & 0.4687 & 0.4761 & 0.4807 & 0.4902 \\
1.5 & 0.5589 & 0.5805 & 0.5927 & 0.6006 & 0.6176 \\
2.0 & 0.6189 & 0.6404 & 0.6546 & 0.6644 & 0.6870 \\
5.0 & 0.6684 & 0.6814 & 0.6942 & 0.7041 & 0.7298 \\
\hline
\end{tabular}

Table 2. The percent relative efficiency of $\hat{\theta}_{\mathrm{MLE}}$ with respect to $\theta_{p}^{* *}$ estimator

\begin{tabular}{rrrrrrr} 
& & \multicolumn{5}{c}{ Sample size } \\
\cline { 3 - 7 } $\boldsymbol{A}$ & $\boldsymbol{p}$ & $\mathbf{1 0}$ & $\mathbf{1 5}$ & $\mathbf{2 0}$ & $\mathbf{2 5}$ & $\mathbf{5 0}$ \\
\hline 0.625 & -1.0 & 112.3948 & 108.2524 & 106.1851 & 104.9460 & 102.4709 \\
& -0.5 & 102.4065 & 101.5986 & 101.1968 & 100.9564 & 100.4771 \\
& 0.0 & 100.0000 & 100.0000 & 100.0000 & 100.0000 & 100.0000 \\
& 0.5 & 110.2408 & 106.6603 & 104.9338 & 103.9179 & 101.9301 \\
& 1.0 & 105.6803 & 104.6816 & 103.8287 & 103.2098 & 101.7449 \\
& 1.5 & 66.3761 & 74.9265 & 80.0713 & 83.4819 & 91.1228 \\
& 2.0 & 36.3377 & 43.3610 & 49.2513 & 54.1038 & 69.0787 \\
& 2.5 & 22.6909 & 25.7553 & 29.2873 & 32.6959 & 46.2440 \\
2.500 & & & & & & \\
& -1.0 & 118.0488 & 111.9126 & 108.8889 & 107.0891 & 103.5223 \\
& -0.5 & 102.4899 & 101.6536 & 101.2378 & 100.9890 & 100.4933 \\
& 0.0 & 100.0000 & 100.0000 & 100.0000 & 100.0000 & 100.0000 \\
& 0.5 & 112.9021 & 108.2732 & 106.0883 & 104.8161 & 102.3553 \\
& 1.0 & 135.1351 & 122.2826 & 116.2791 & 112.8158 & 106.2022 \\
& 1.5 & 133.7216 & 124.6512 & 119.0925 & 115.5048 & 107.9158 \\
& 2.0 & 98.2897 & 101.5389 & 102.6273 & 102.9314 & 102.4205 \\
& 2.5 & 67.5687 & 71.7393 & 75.8728 & 79.1579 & 87.8875 \\
\hline
\end{tabular}

Table 2 (continued). 


\section{SHRINKAGE ESTIMATORS FOR RAYLEIGH DISTRIBUTION}

\begin{tabular}{rrrrrrr} 
& & \multicolumn{5}{c}{ Sample size } \\
\cline { 3 - 7 } $\boldsymbol{\lambda}$ & $\boldsymbol{p}$ & $\mathbf{1 0}$ & $\mathbf{1 5}$ & $\mathbf{2 0}$ & $\mathbf{2 5}$ & $\mathbf{5 0}$ \\
\hline 5.000 & -1.0 & 121.0000 & 113.7778 & 110.2500 & 108.1600 & 104.0400 \\
& -0.5 & 102.5305 & 101.6803 & 101.2577 & 101.0049 & 100.5012 \\
& 0.0 & 100.0000 & 100.0000 & 100.0000 & 100.0000 & 100.0000 \\
& 0.5 & 114.2392 & 109.0729 & 106.6572 & 105.2573 & 102.5627 \\
& 1.0 & 156.2500 & 133.1361 & 123.4568 & 118.1474 & 108.5069 \\
& 1.5 & 263.1945 & 183.7882 & 155.9376 & 141.8949 & 118.5045 \\
& 2.0 & 566.8934 & 290.5475 & 216.2630 & 183.0105 & 133.7114 \\
& 2.5 & 1644.9660 & 533.9921 & 331.6380 & 254.5150 & 155.9988 \\
7.500 & & & & & & \\
& -1.0 & 118.0488 & 111.9126 & 108.8889 & 107.0891 & 103.5224 \\
& -0.5 & 102.4899 & 101.6536 & 101.2378 & 100.9890 & 100.4933 \\
& 0.0 & 100.0000 & 100.0000 & 100.0000 & 100.0000 & 100.0000 \\
& 0.5 & 112.9021 & 108.2732 & 106.0883 & 104.8161 & 102.3553 \\
& 1.0 & 135.1351 & 122.2826 & 116.2791 & 112.8158 & 106.2022 \\
& 1.5 & 133.7216 & 124.6512 & 119.0925 & 115.5048 & 107.9158 \\
& 2.0 & 98.2897 & 101.5389 & 102.6273 & 102.9314 & 102.4205 \\
& 2.5 & 67.5687 & 71.7393 & 75.8728 & 79.1579 & 87.8875 \\
& & & & & & \\
& -1.0 & 98.7755 & 98.9372 & 99.1011 & 99.2293 & 99.5598 \\
& -0.5 & 102.1671 & 101.4407 & 101.0790 & 100.8624 & 100.4305 \\
& 0.0 & 100.0000 & 100.0000 & 100.0000 & 100.0000 & 100.0000 \\
& 0.5 & 103.2356 & 102.2745 & 101.7465 & 101.4158 & 100.7256 \\
& 1.0 & 64.9350 & 74.0131 & 79.3650 & 82.8912 & 90.7770 \\
& 1.5 & 27.0942 & 34.8759 & 41.2049 & 46.4272 & 62.9312 \\
& 2.0 & 12.9108 & 16.3661 & 19.7223 & 22.8709 & 35.6598 \\
& 2.5 & 7.7921 & 9.0520 & 10.5823 & 12.1559 & 19.5613 \\
\hline
\end{tabular}

Here the relative efficiencies of the defined estimator classes with respect to the unbiased estimator of the scale parameter of the Rayleigh distribution is calculated using the different values of $n, p$, and $\lambda$. The values of $\lambda$ are selected by considering the efficiency range for large sample size values in numerical studies. The percent relative efficiency of $\theta_{p}^{* *}$ estimator with respect to $\hat{\theta}_{\mathrm{MLE}}$ is calculated for the different values of $n, p$, and $\lambda$ with the help of Equation (19). These calculated values are summarized in Table 2 . The $\theta_{p}^{* *}$ estimator performed better as compared to the unbiased estimator whenever the value of $p$ was between -1 and 1 , for each value of $\lambda$ and $n$, while increased $p$ values cause a decrease in the efficiency of the proposed biased estimator class with respect to the unbiased estimator.

Table 3. The percent relative efficiency of $\hat{\theta}_{\text {MLE }}$ with respect to $\theta_{p}^{*}$ 


\section{OMER ET AL}

\begin{tabular}{|c|c|c|c|c|c|c|}
\hline \multirow[b]{2}{*}{$\lambda$} & \multirow[b]{2}{*}{$p$} & \multicolumn{5}{|c|}{ Sample size } \\
\hline & & 10 & 15 & 20 & 25 & 50 \\
\hline \multirow[t]{8}{*}{0.625} & -1.0 & 100.1629 & 100.1627 & 100.1624 & 100.1622 & 100.1609 \\
\hline & -0.5 & 100.0502 & 100.0502 & 100.0501 & 100.0501 & 100.0500 \\
\hline & 0.0 & 100.0000 & 100.0000 & 100.0000 & 100.0000 & 100.0000 \\
\hline & 0.5 & 100.0098 & 100.0098 & 100.0098 & 100.0098 & 100.0098 \\
\hline & 1.0 & 100.0768 & 100.0768 & 100.0767 & 100.0767 & 100.0764 \\
\hline & 1.5 & 100.1982 & 100.1978 & 100.1975 & 100.1971 & 100.1952 \\
\hline & 2.0 & 100.3710 & 100.3697 & 100.3683 & 100.3670 & 100.3603 \\
\hline & 2.5 & 100.5922 & 100.5888 & 100.5853 & 100.5819 & 100.5647 \\
\hline \multirow[t]{8}{*}{2.500} & -1.0 & 100.1633 & 100.1632 & 100.1631 & 100.1630 & 100.1626 \\
\hline & -0.5 & 100.0502 & 100.0502 & 100.0502 & 100.0502 & 100.0502 \\
\hline & 0.0 & 100.0000 & 100.0000 & 100.0000 & 100.0000 & 100.0000 \\
\hline & 0.5 & 100.0098 & 100.0098 & 100.0098 & 100.0098 & 100.0098 \\
\hline & 1.0 & 100.0769 & 100.0769 & 100.0769 & 100.0768 & 100.0768 \\
\hline & 1.5 & 100.1987 & 100.1986 & 100.1985 & 100.1984 & 100.1977 \\
\hline & 2.0 & 100.3728 & 100.3724 & 100.3719 & 100.3715 & 100.3693 \\
\hline & 2.5 & 100.5969 & 100.5957 & 100.5946 & 100.5935 & 100.5879 \\
\hline \multirow[t]{8}{*}{5.000} & -1.0 & 100.1635 & 100.1635 & 100.1635 & 100.1635 & 100.1635 \\
\hline & -0.5 & 100.0502 & 100.0502 & 100.0502 & 100.0502 & 100.0502 \\
\hline & 0.0 & 100.0000 & 100.0000 & 100.0000 & 100.0000 & 100.0000 \\
\hline & 0.5 & 100.0098 & 100.0098 & 100.0098 & 100.0098 & 100.0098 \\
\hline & 1.0 & 100.0769 & 100.0769 & 100.0769 & 100.0769 & 100.0769 \\
\hline & 1.5 & 100.1990 & 100.1990 & 100.1990 & 100.1991 & 100.1990 \\
\hline & 2.0 & 100.3737 & 100.3737 & 100.3737 & 100.3737 & 100.3737 \\
\hline & 2.5 & 100.5991 & 100.5991 & 100.5991 & 100.5991 & 100.5991 \\
\hline \multirow[t]{8}{*}{7.500} & -1.0 & 100.1633 & 100.1632 & 100.1631 & 100.1630 & 100.1626 \\
\hline & -0.5 & 100.0502 & 100.0502 & 100.0502 & 100.0502 & 100.0502 \\
\hline & 0.0 & 100.0000 & 100.0000 & 100.0000 & 100.0000 & 100.0000 \\
\hline & 0.5 & 100.0098 & 100.0098 & 100.0098 & 100.0098 & 100.0098 \\
\hline & 1.0 & 100.0769 & 100.0769 & 100.0769 & 100.0768 & 100.0768 \\
\hline & 1.5 & 100.1987 & 100.1986 & 100.1985 & 100.1984 & 100.1977 \\
\hline & 2.0 & 100.3728 & 100.3724 & 100.3719 & 100.3715 & 100.3693 \\
\hline & 2.5 & 100.5969 & 100.5957 & 100.5946 & 100.5935 & 100.5879 \\
\hline \multirow[t]{8}{*}{12.500} & -1.0 & 100.1620 & 100.1612 & 100.1605 & 100.1597 & 100.1559 \\
\hline & -0.5 & 100.0501 & 100.0500 & 100.0499 & 100.0499 & 100.0495 \\
\hline & 0.0 & 100.0000 & 100.0000 & 100.0000 & 100.0000 & 100.0000 \\
\hline & 0.5 & 100.0098 & 100.0098 & 100.0098 & 100.0098 & 100.0098 \\
\hline & 1.0 & 100.0766 & 100.0764 & 100.0763 & 100.0761 & 100.0753 \\
\hline & 1.5 & 100.1967 & 100.1956 & 100.1945 & 100.1934 & 100.1878 \\
\hline & 2.0 & 100.3658 & 100.3619 & 100.3580 & 100.3540 & 100.3344 \\
\hline & 2.5 & 100.5789 & 100.5687 & 100.5586 & 100.5485 & 100.4980 \\
\hline
\end{tabular}




\section{SHRINKAGE ESTIMATORS FOR RAYLEIGH DISTRIBUTION}

Similarly, the relative efficiency of the proposed class estimator $\theta_{p}^{*}$ with

respect to $\hat{\theta}_{\mathrm{MLE}}$ is computed for different values of $n, p$, and $\lambda$. These computed values are given in Table 3. It can also see that the proposed class estimator $\theta_{p}^{*}$ performs better for each value of $n, p$, and $\lambda$ as compared to the unbiased estimator. Moreover, when the value of $\lambda$ is 7 the proposed class of estimator $\theta_{p}^{*}$ performs similar to $\hat{\theta}_{\mathrm{MLE}}$. Furthermore, when the estimator $\theta_{p}^{* *}$ given in Table 2 is compared to the estimator given in Table 3, it is detected that the efficiency range of the estimator class $\theta_{p}^{* *}$ with respect to the estimator class $\theta_{p}^{*}$ is larger than that of $\hat{\theta}_{\mathrm{MLE}}$. It is also observed that the proposed class of shrinkage estimators given in Equation (6) performs better than the traditional MLE for each value of $n$ and $p$. However, the percent relative efficiency of the proposed class of biased estimators is symmetric around $\lambda=5$ for a given $p$ and $n$. It shows that the proposed class of estimators performs well even when the prior guess $\theta_{0}$ is away from the true but unknown value of the parameter $\theta$. Also, the relative efficiency of this class of estimators is not much higher. Meanwhile, the proposed class of estimators given in Equation (20) performs well compared to the usual MLE but its performance depends upon the values of $n, p$, and $\lambda$. For smaller values of $n$ and $p$, it performs better at a given $\lambda$. As the sample size increases, $\lambda$ tends to away from 5 , and its performance deteriorates and becomes very poor when sample size, $p$ and $\lambda$ are larger. It is observed that the proposed class of estimators can be made highly efficient by taking $p=2,2.5$ when sample size is around 20 and $\lambda$ is expected to be approximately 5. Moreover, it is observed that, in general, $p$ should be in the interval $(-1,1)$ so that the proposed class of estimators may be fruitfully used. Above discussion establishes the superiority of the proposed classes of biased estimators.

\section{Simulation}

A random sample was generated with different sample sizes from the Rayleigh distribution with $\theta=5$ and then calculated the unbiased and biased estimators from the generated samples. The procedure is iterated 10000 times. The MSE of both estimators is calculated to judge the performance of the proposed estimators. We considered $n=10,20$, and 50 and $p=-1.00$ to 2.50 with an interval of 0.50 . The simulated results are shown in Table 4 . It can be seen that the proposed estimator $\theta_{p}^{*}$ performs well compared to the estimator $\theta_{p}^{* *}$ when the value of $p$ lies in the 


\section{OMER ET AL}

interval $(-1 \leq p \leq 0)$ for each value of $\lambda$. Furthermore, the percent relative efficiency of both estimators decreased when sample size increases. It is evident from Table 4 that the estimator $\theta_{p}^{*}$ performs much better than the estimator $\theta_{p}^{* *}$ and these results are in agreement with the theoretical results.

Table 4. The percent relative efficiency of the estimators $\theta_{p}^{*}$ and $\theta_{p}^{* *}$ with respect to $\hat{\theta}_{\mathrm{MLE}}$

\begin{tabular}{|c|c|c|c|c|c|c|c|}
\hline \multirow[b]{3}{*}{$\lambda$} & \multirow[b]{3}{*}{$p$} & \multicolumn{6}{|c|}{ Sample size } \\
\hline & & \multicolumn{2}{|c|}{10} & \multicolumn{2}{|c|}{20} & \multicolumn{2}{|c|}{50} \\
\hline & & $\theta_{p}^{*}$ & $\theta_{p}^{* *}$ & $\theta_{p}^{*}$ & $\boldsymbol{\theta}_{p}^{* *}$ & $\theta_{p}^{*}$ & $\theta_{p}^{* *}$ \\
\hline \multirow[t]{8}{*}{0.5} & -1.0 & 134.8489 & 117.9759 & 116.1170 & 108.8182 & 105.9455 & 103.3986 \\
\hline & -0.5 & 113.0125 & 102.5095 & 106.0271 & 101.2272 & 102.4421 & 100.5107 \\
\hline & 0.0 & 100.0000 & 100.0000 & 100.0000 & 100.0000 & 100.0000 & 100.0000 \\
\hline & 0.5 & 102.4252 & 112.4855 & 101.2368 & 106.0785 & 100.4898 & 102.3402 \\
\hline & 1.0 & 117.9180 & 134.8382 & 108.9946 & 116.5100 & 103.5303 & 106.2363 \\
\hline & 1.5 & 135.0329 & 131.6477 & 116.9538 & 117.2962 & 107.4184 & 108.4199 \\
\hline & 2.0 & 137.1994 & 99.3177 & 118.5453 & 102.8011 & 107.1770 & 102.0707 \\
\hline & 2.5 & 113.9579 & 67.8402 & 104.8259 & 76.1585 & 100.7715 & 88.3023 \\
\hline \multirow[t]{8}{*}{1.0} & -1.0 & 156.2500 & 121.0000 & 123.4568 & 110.2500 & 108.5069 & 104.0400 \\
\hline & -0.5 & 114.2392 & 102.5304 & 106.6572 & 101.2577 & 102.5628 & 100.5012 \\
\hline & 0.0 & 100.0000 & 100.0000 & 100.0000 & 100.0000 & 100.0000 & 100.0000 \\
\hline & 0.5 & 102.5304 & 114.2392 & 101.2577 & 106.6572 & 100.5012 & 102.5628 \\
\hline & 1.0 & 121.0000 & 156.2500 & 110.2500 & 123.4568 & 104.0400 & 108.5069 \\
\hline & 1.5 & 162.0400 & 263.1945 & 128.5714 & 155.9376 & 110.8653 & 118.5045 \\
\hline & 2.0 & 243.3600 & 566.8934 & 160.0225 & 216.2630 & 121.5286 & 133.7115 \\
\hline & 2.5 & 405.8534 & 1644.9660 & 211.8779 & 331.6380 & 136.9558 & 155.9989 \\
\hline \multirow[t]{8}{*}{1.5} & -1.0 & 134.3012 & 117.7642 & 116.3531 & 108.9283 & 106.7376 & 103.7642 \\
\hline & -0.5 & 113.0051 & 102.5049 & 105.9939 & 101.2203 & 102.4261 & 100.5078 \\
\hline & 0.0 & 100.0000 & 100.0000 & 100.0000 & 100.0000 & 100.0000 & 100.0000 \\
\hline & 0.5 & 102.4557 & 112.7095 & 101.1636 & 105.6888 & 100.4870 & 102.3230 \\
\hline & 1.0 & 118.2849 & 135.4221 & 109.0479 & 116.6330 & 103.5407 & 106.2374 \\
\hline & 1.5 & 137.1121 & 134.3306 & 117.6372 & 118.6150 & 107.5710 & 108.5778 \\
\hline & 2.0 & 138.1145 & 99.2876 & 119.0729 & 103.3666 & 108.3324 & 103.5204 \\
\hline & 2.5 & 115.3105 & 67.9699 & 102.1367 & 74.0475 & 100.9496 & 88.2497 \\
\hline \multirow[t]{8}{*}{2.0} & -1.0 & 96.4793 & 110.2760 & 98.2127 & 104.5576 & 99.9544 & 102.0701 \\
\hline & -0.5 & 109.0627 & 102.3512 & 104.8835 & 101.2702 & 101.6787 & 100.4558 \\
\hline & 0.0 & 100.0000 & 100.0000 & 100.0000 & 100.0000 & 100.0000 & 100.0000 \\
\hline & 0.5 & 102.4054 & 109.3374 & 101.1150 & 104.0505 & 100.4903 & 101.8299 \\
\hline & 1.0 & 110.0865 & 96.2502 & 105.3312 & 99.6157 & 101.3720 & 98.5737 \\
\hline & 1.5 & 93.0756 & 53.9501 & 95.9266 & 70.9762 & 97.6059 & 85.5818 \\
\hline & 2.0 & 58.9238 & 28.1040 & 65.6110 & 39.6435 & 81.1719 & 61.2328 \\
\hline & 2.5 & 36.4582 & 17.6169 & 40.9252 & 22.8336 & 56.2884 & 38.2200 \\
\hline
\end{tabular}




\section{SHRINKAGE ESTIMATORS FOR RAYLEIGH DISTRIBUTION}

\section{Conclusion}

Sometimes, it may be preferable to use biased estimators given that they have smaller MSE than the variance of the unbiased estimators. Such biased estimators are generally developed using shrinkage estimation techniques. Taking into consideration such case, in this study, two different classes of shrinkage estimators are proposed. The proposed estimators are defined after minimizing the MSEs. To judge the relative performance of the proposed estimators, a numerical and simulation study are conducted where percent relative bias and MSE are considered as performance criteria. As a conclusion, it is stated that in order to have a better estimator when there is a little information about the likely value of the unknown parameter of interest, one should use the proposed class of estimators given in Equation (6). However, if some information is available as a prior guess about the likely values of the parameter, one should use the proposed class of estimators given in Equation (20) and $p$ should be chosen in the vicinity of 0 .

\section{References}

Bhatnagar, S. (1986). On the use of population variance in estimating mean. Journal of the Indian Society of Agricultural Statistics, 38, 403-409.

Das, B. (1975). Estimation of $\mu^{2}$ in normal population. Calcutta Statistical Association Bulletin, 24(1-4), 135-140. doi: 10.1177/0008068319750115

Ebegil, M., \& Ozdemir, S. (2016). Two different shrinkage estimator classes for the shape parameter of classical Pareto distribution. Hacettepe Journal of Mathematics and Statistics, 45(4), 1231-1244. doi: 10.15672/HJMS.20158812908

Jani, P. (1991). A class of shrinkage estimators for the scale parameter of the exponential distribution. IEEE Transactions on Reliability, 40(1), 68-70. doi: $10.1109 / 24.75337$

Kourouklis, S. (1994). Estimation in the 2-parameter exponential distribution with prior information. IEEE Transactions on Reliability, 43(3), 446450. doi: 10.1109/24.326444

Mehta, J. S., \& Srinivasan, R. (1971). Estimation of the mean by shrinkage to a point. Journal of the American Statistical Association, 66(333), 86-90. doi: 10.1080/01621459.1971.10482224

Prakash, G. (2009). Some estimators for the Pareto distribution. Journal of Scientific Research, 1(2), 236-247. doi: 10.3329/jsr.v1i2.1642 


\section{OMER ET AL}

Prakash, G., Singh, D., \& Singh, R. (2006). Some test estimators for the scale parameter of classical pareto distribution. Journal of Statistical Research, 40(2), 41-54. Retrieved from http://jsr.isrt.ac.bd/article/test-estimators-scaleparameter-classical-pareto-distribution/

Rao, V., \& Singh, J. (1982). A note on estimation of $\mu^{2}$ in normal density. Journal of the Indian Society of Agricultural Statistics, 34(1), 82-84.

Rayleigh, L. (1880). On the resultant of a large number of vibrations of the same pitch and of arbitrary phase. The London, Edinburgh, and Dublin Philosophical Magazine and Journal of Science Series 5, 10(60), 73-78. doi: 10.1080/14786448008626893

Rytgaard, M. (1990). Estimation in the Pareto distribution. ASTIN Bulletin, 20(2), 201-216. doi: 10.2143/AST.20.2.2005443

Searls, D. T. (1964). The utilization of a known coefficient of variation in the estimation procedure. Journal of the American Statistical Association, 59(308),1225-1226. doi: 10.1080/01621459.1964.10480765

Singh, H., \& Katyar, N. (1988). A generalized class of estimators for common parameters of two normal distribution with known coefficient of variation. Journal of the Indian Society of Agricultural Statistics, 40(2), 127-149.

Singh, H. P., \& Saxena, S. (2003). A class of shrinkage estimators for variance of a normal population. Brazilian Journal of Probability and Statistics, 17(1), 41-56.

Singh, H. P., \& Shukla, S. K. (2003). A family of shrinkage estimators for the square of mean in normal distribution. Statistical Papers, 44(3), 433-442. doi: 10.1007/s00362-003-0165-8

Singh, H. P., \& Singh, R. (1997). A class of shrinkage estimators for the variance of a normal population. Microelectronics Reliability, 37(5), 863-867. doi: 10.1016/S0026-2714(96)00103-5

Srivastava, V., Dwivedi, T., \& Bhatnagar, S. (1980). Estimation of the square of mean in normal population. Statistica, 40(4), 455-466.

Thompson, J. R. (1968). Some shrinkage techniques for estimating the mean. Journal of the American Statistical Association, 63(321), 113-122. doi: 10.1080/01621459.1968.11009226 